\title{
HEXITEC: A High Energy X-Ray Spectroscopic Imaging Detector for Synchrotron Applications
}

\author{
M. C. Veale ${ }^{1 *}$, P. Seller ${ }^{1}$, M. Wilson ${ }^{1}$ and E. Liotti ${ }^{2}$ \\ 1. UKRI Science \& Technology Facilities Council, Rutherford Appleton Laboratory, OX11 0QX. \\ 2. Department of Materials, University of Oxford, Oxford OX1 3PH
}

\begin{abstract}
HEXITEC is a compact high energy X-ray spectroscopic imaging detector system developed by the STFC Rutherford Appleton Laboratory (UK). The system is based on the high density compound semiconductor cadmium telluride and consists of an $80 \times 80$ array of pixels on a $250 \mu \mathrm{m}$ pitch with each channel capable of measuring X-ray spectra in the energy range $2-200 \mathrm{keV}$ with an energy resolution of $800 \mathrm{eV}$. The system operates at room temperature and removes the need for large cryogenic cooling systems used by other systems covering similar energy ranges. The system has applications at synchrotrons in experiments where mechanical, structural or chemical information is required from thick samples or to determine the chemical composition of samples containing multiple high $\mathrm{Z}$ materials. In this article the operation of the system is described, the sensor performance characterised and a simple example of spectroscopic imaging at a synchrotron beamline demonstrated.
\end{abstract}

\section{Introduction}

Over the last decade, the High Energy X-Ray Imaging Technology (HEXITEC) detector system for spectroscopic imaging of hard X-rays and $\gamma$-rays has been developed by the Science \& Technology Facilities Council (STFC). The system is based upon the room-temperature compound semiconductor cadmium telluride (CdTe) which, due to its high density and large band gap, allows the measurement of X-ray and $\gamma$-ray spectra up to energies of $200 \mathrm{keV}$ with an energy resolution of $<1 \mathrm{keV}$ without the need for cryogenic cooling systems [1]. The technology differs significantly from single photon counting detector systems where a count is registered each time the output of a pixel is above a threshold. In the HEXITEC system each $250 \mu \mathrm{m}$ pixel is able to record the precise energy deposited by an interaction and, over multiple frames, compiles energy-resolved $\mathrm{X}$-ray spectra on a per pixel basis rather than a simple 2D image. The system has been used at synchrotrons in applications which wish to discern mechanical, structural or chemical information from within thick samples or to determine the chemical composition of samples containing multiple high $\mathrm{Z}$ materials which, typically, have closely spaced K-line X-ray emissions. While originally developed for materials science applications [2], [3], the system has been successfully used across a diverse range of fields including pure science [4], [5], solar physics [6], medical imaging [7] and security applications [8]. This article will review the current status of the technology, the performance of the latest system and demonstrates the use of the system on a synchrotron beamline.

\section{The HEXITEC Detector System}

The heart of the system is the HEXITEC application specific integrated circuit (ASIC) which consists of 6,400 channels in an $80 \times 80$ array on a $250 \mu \mathrm{m}$ pitch [9]. Each pixel of the ASIC contains a charge sensitive preamplifier, leakage current compensation circuit, shaping amplifier and peak-track-andhold circuit. The system also makes use of cadmium telluride sensors produced by Acrorad Ltd [10] which have dimensions of $20.45 \times 20.35 \times 1.00 \mathrm{~mm}^{3}$. Sensors have a large planar platinum cathode and a pixelated aluminium anode with a pad size of $200 \mu \mathrm{m}$ and an inter-pixel gap of $50 \mu \mathrm{m}$. The sensor is bonded directly to the ASIC using a gold stud and silver-loaded epoxy low temperature process developed at STFC [11]. The bonded devices are mounted on an aluminium module and the I/O pads of the ASIC wire bonded to a readout PCB that is fixed to the module, see Figure 1 (b). This modular design allows the production of the standard single module HEXITEC system discussed here as well as larger $\mathrm{N} \times \mathrm{N}$ systems [12]. 
When an X-ray or $\gamma$-ray interacts in the CdTe sensor it creates electrons and holes which, under the influence of the applied bias voltage, drift towards the detector electrodes. In HEXITEC, and in the majority of CdTe-based detectors, it is the electrons that form the detector signal as their charge transport properties are significantly better than those of holes [13]. As the electrons drift away from the planar cathode they induce a mirror charge on the pixelated anode as described by the ShockleyRamo theorem [14]. This mirror charge is amplified by the pixel's charge sensitive preamplifier and is then filtered for high frequency noise by a CR-RC shaper $(\tau \sim 2 \mu \mathrm{s})$. A peak-hold circuit in each pixel tracks the output of the shaper and holds the maximum magnitude measured during the frame time until readout. The magnitude of the peak-hold output is directly proportional to the charge induced on the pixel which in turn is proportional to the energy of the original interaction. The analogue voltages stored in each pixel are read out using a rolling shutter operating at a maximum frame rate of $8.9 \mathrm{kHz}$, these are then multiplexed off of the ASIC to 4 ADCs that digitize the voltages. The data is then packaged locally in an FPGA before transmission to the control PC over a GigE Ethernet connection.

The current single module HEXITEC DAQ system can be seen in Figure 1 (a). As well as containing the detector and the associated readout electronics, the DAQ also contains the high voltage source that is required to bias the CdTe sensor $(-500 \mathrm{~V})$ as well as a Peltier-based temperature control system that holds the ASIC at a stable temperature $\left(+/-1^{\circ} \mathrm{C}\right)$. All that is required to run the system is a $12 \mathrm{~V}$ power supply, Ethernet connection to a control PC and the HEXITEC software which is used to control the system as well as providing 'on-the-fly' spectroscopy during acquisitions. The total size of the entire HEXITEC DAQ is $22 \mathrm{~cm} \times 6 \mathrm{~cm} \times 6 \mathrm{~cm}$.
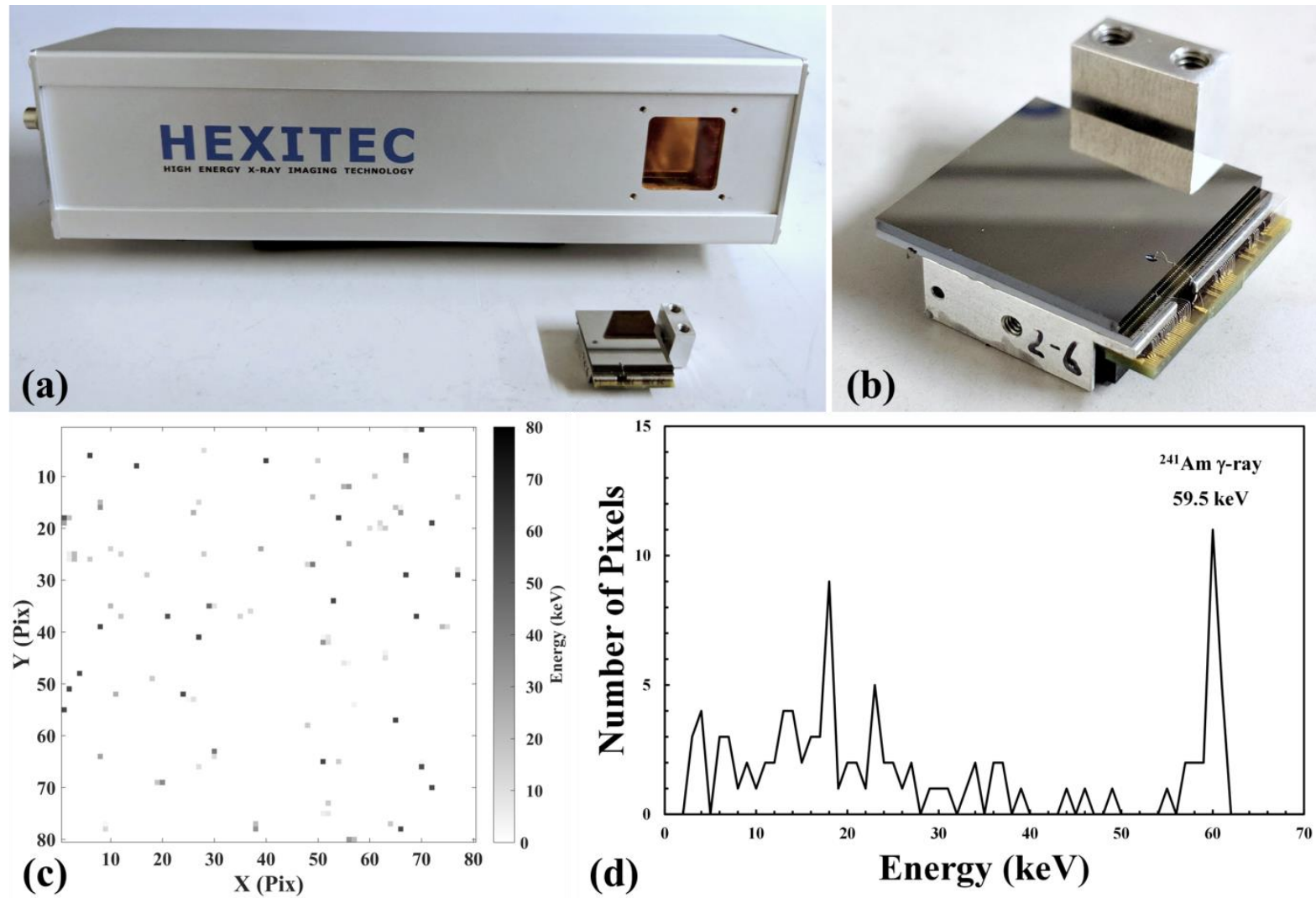

Figure 1: (a) The latest generation of the HEXITEC 'GigE' data acquisition system, (b) an individual $20 \mathrm{~mm} \times 20 \mathrm{~mm} \times 1 \mathrm{~mm}$ CdTe HEXITEC module, (c) a single frame of spectroscopic data recorded with an ${ }^{241} \mathrm{Am}$ sealed source and (d) a histogram of the energy per pixel in the single frame. 


\section{The Spectroscopic Performance of the HEXITEC System}

To characterise the performance of the HEXITEC system, spectroscopic data was recorded with an

${ }^{241} \mathrm{Am}$ sealed source, principle emission at $60 \mathrm{keV}$, followed by a ${ }^{57} \mathrm{Co}$ sealed source, principle emissions $122 \mathrm{keV}$ and $136 \mathrm{keV}$. A bias voltage of $-500 \mathrm{~V}$ was applied across the CdTe detector and the temperature held at $28+/-1{ }^{\circ} \mathrm{C}$. The sealed sources were placed at a distance of $10 \mathrm{~cm}$ from the detector to ensure a flat field irradiation across the entire sensor. Figure 1 (c) shows an example of a single frame of data corresponding to an integration time of $\sim 100 \mu \mathrm{s}$. This frame of data contains 100 pixels that are above the low energy threshold of $2 \mathrm{keV}$ corresponding to an occupancy of $\sim 1.6 \%$. If the energy values recorded by each of the hit pixels are compiled in a histogram then, from just a single frame of data, it is possible to begin to identify the principle emission of the ${ }^{241} \mathrm{Am}$ source, see Figure 1 (d).

As the percentage of hit pixels is relatively low, it is possible to identify those events that occur in a single pixel but also the relatively large number that involve more than one pixel. These events that occur over multiple pixels demonstrate the phenomena of charge sharing which occurs in small pixel detectors like HEXITEC [15]. Typically, the charge cloud created by an X-ray interaction in CdTe will have an initial size of $\sim 10 \mu \mathrm{m}$ and this increases due to Einsteinian diffusion as the charge moves from the point of interaction to the collecting electrodes. As the size of the inter-pixel gap is similar to the size of the charge clouds being measured, there is a strong chance of sharing of charge between pixels due to the detector geometry. In addition to this geometrical charge sharing, if the X-ray energy exceeds $\sim 25 \mathrm{keV}$ then there is a significant chance of generating $\mathrm{Cd}$ and Te florescence $\mathrm{X}$-rays from the detector material itself. These florescence $X$-rays can have mean free paths in the detector of the order $100 \mu \mathrm{m}$ and result in an increased probability of charge sharing at these energies. Where charge sharing does occur, if uncorrected, it results in an excess of low energy events that can degrade the spectroscopic performance of the detector.

Figure 2 (Left) shows a typical X-ray spectrum collected by a single pixel collected over a 10 minute acquisition $\left(\sim 10^{6}\right.$ frames $)$ before and after correction for charge sharing. Without correction, all of the energy values recorded by the pixel are histogrammed for all frames producing the 'RAW' spectrum. Alternatively, a charge sharing discrimination (CSD) algorithm can be used to remove those events that involve multiple pixels. To apply the correction, each recorded frame of data is inspected in regions of $3 \times 3$ pixels for neighbouring pixels that have measured an energy above the $2 \mathrm{keV}$ low energy threshold. If neighbouring pixels do show a signal then the energies they have recorded are not included in the data analysis. Figure 2 (left) demonstrates how the removal of these shared events greatly reduces the low energy background seen in the RAW spectrum. The effect of the CSD processing can be quantified through the peak-to-valley ratio of the $60 \mathrm{keV}^{241} \mathrm{Am}$ peak which has a median value of 1.8 for the RAW spectra but increases to 12.2 after CSD correction. It is worth noting that all corrections are carried out in post-processing enabling the user to select precisely how they wish to present the data, no information is lost. Figure 2 (Right) demonstrates how the application of the correction has no effect on the uniformity of the detector with the number of $60 \mathrm{keV}$ counts varying by $\sim 5 \%$ from pixel to pixel. Some larger variation can be seen at the detector periphery where the lower resistance edges of the CdTe crystal [16] reduce the performance of these pixels.

While the use of a charge sharing algorithm improves the resolution of the processed spectra, it does result in a reduction in the number of events included in the analysis. Figure 3 (Left) shows the distribution of the number of pixels that are involved in a single interaction in the detector at $60 \mathrm{keV}$ and $122 \mathrm{keV}$. At the lower energy $54.8 \%$ of all events involve more than one pixel while at the higher energy a larger initial charge cloud leads to a slight increase in the total amount of charge sharing to $55.6 \%$ as well as an increase in the number of interactions involving $>2$ pixels. If maintaining the highest possible counting efficiency is essential for a particular application then it is possible to recover the total energy deposited in a shared event by using a charge sharing addition algorithm although this does impact on the spectroscopic performance of the detector [15]. As all spectra are produced in post-processing, a combination of both these techniques can be used to maximise the 
information contained within any data set. It is worth noting that the ability to correct for charge sharing requires the occupancy, the percentage of pixel showing a hit, of a single frame of data to not exceed $\sim 11 \%$ i.e. no more than one event in a block of $3 \times 3$ pixels. For this reason, the optimal spectroscopic performance of the HEXITEC system is achieved when X-ray fluxes are limited to < $10^{6}$ photons $\mathrm{s}^{-1} \mathrm{~cm}^{-2}$.
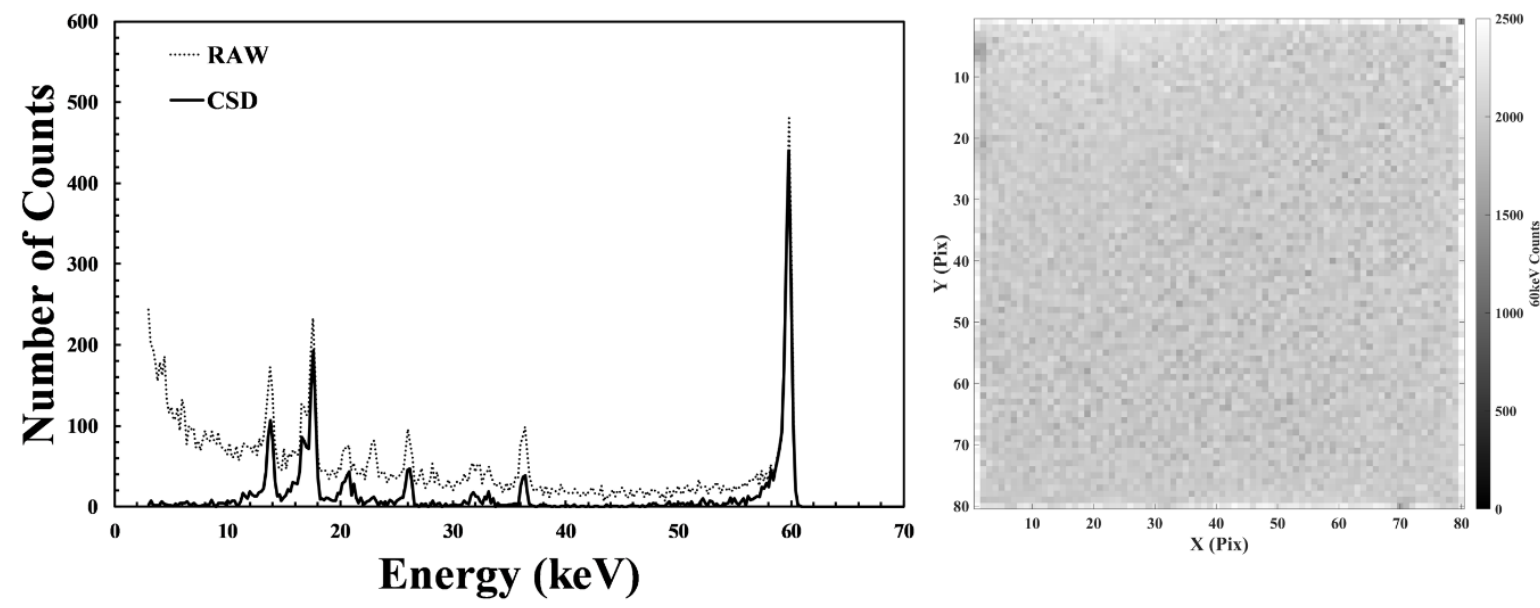

Figure 2: (Left) An example of a charge sharing discriminated (CSD) single pixel spectrum recorded with the detector. (Right) A map of the total number of ${ }^{241} \mathrm{Am} 60 \mathrm{keV} \gamma$-rays recorded per pixel during a flat field irradiation after CSD correction.
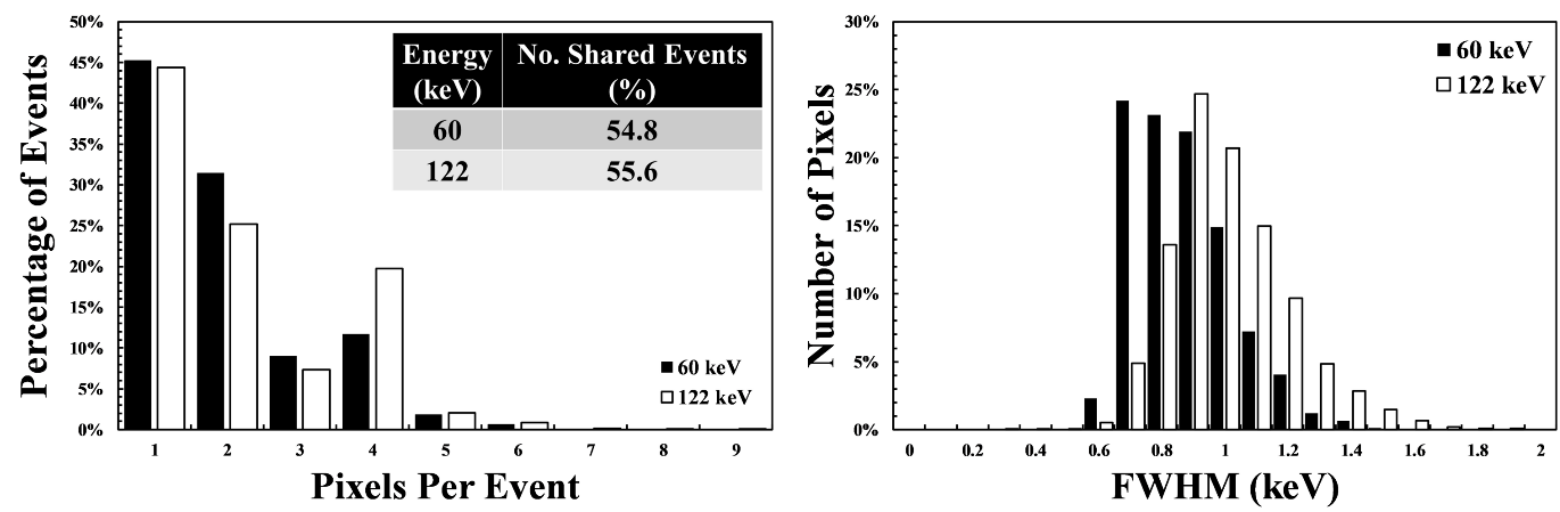

Figure 3: (Left) A histogram of the number of pixels involved in an individual interaction in the detector at $60 \mathrm{keV}$ and $122 \mathrm{keV}$. (Right) The distribution of FWHM per pixel measured at $60 \mathrm{keV}$ and $122 \mathrm{keV}$.

The uniformity of the detector response can be quantified from the FWHM of the main photo-peaks measured for each pixel. Figure 3 (Right) shows the distribution of FWHM values measured by each pixel at $60 \mathrm{keV}$ and $122 \mathrm{keV}$ which have average values of $0.81+/-0.14 \mathrm{keV}$ and $0.93+/ 0.19 \mathrm{keV}$ respectively. The small increase in FWHM measured at the higher energy maybe a result of an increased depth of interaction of these photons in the sensor. At $60 \mathrm{keV}$ the mean free path of a $\gamma$-ray is $0.26 \mathrm{~mm}$ while at $122 \mathrm{keV}$ it is $1.70 \mathrm{~mm}$, as the sensor is only $1 \mathrm{~mm}$ thick, this means that the higher energy $\gamma$-rays are capable of depositing energy at any depth in the sensor. Those events that occur close to the pixelated anode will suffer from a larger contribution from the hole charge carrier leading to greater low-energy tailing and a reduction in spectroscopic performance [17]. 

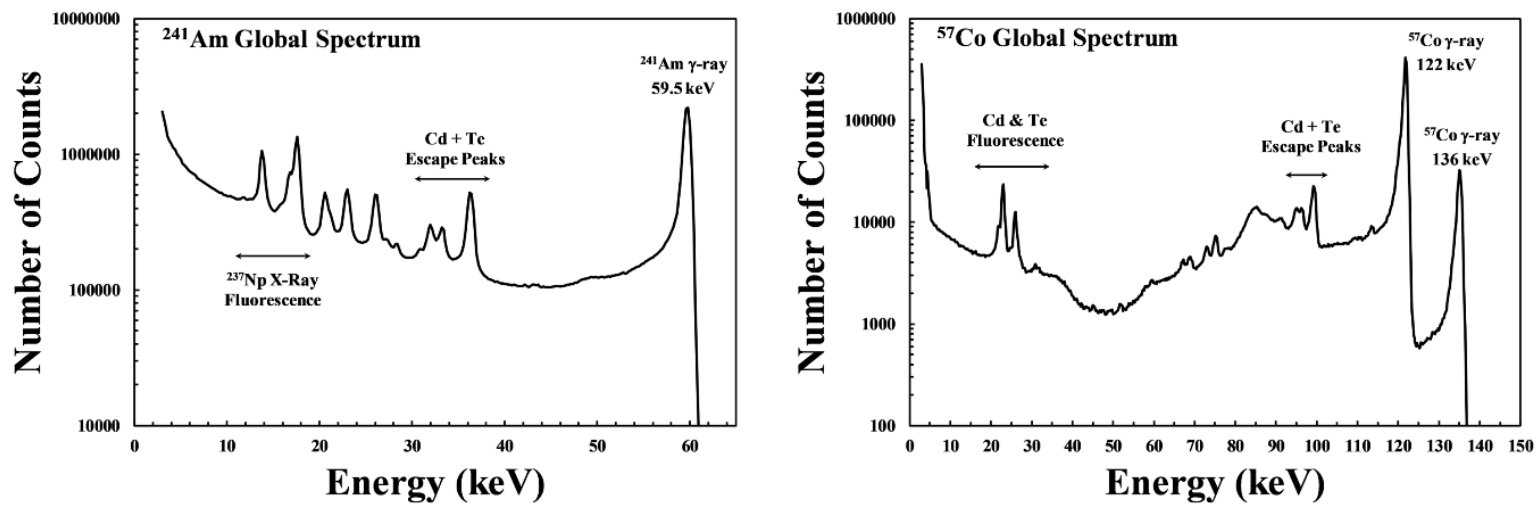

Figure 4: (Left) The ${ }^{241} \mathrm{Am}$ global CSD spectrum recorded summed across all of the 6,400 channels of the detector. (Right) The global CSD spectrum recorded with a ${ }^{57} \mathrm{Co}$ sealed source. The spectra are plotted on a $\mathrm{LOG}_{10}$ scale.

The CSD spectrum recorded by each of the 6,400 channels of the detector can be summed together to produce a single global spectrum which also demonstrates the excellent uniformity of the sensors. Figure 3 (left) shows the global spectrum recorded with the ${ }^{241} \mathrm{Am}$ sealed source which has a global FWHM of $1.2 \mathrm{keV}$. As well as the main $60 \mathrm{keV}$ photopeak, prominent peaks are also observed at 14 $\mathrm{keV}$ and $18 \mathrm{keV} \mathrm{L}_{\alpha}$ and $\mathrm{L}_{\beta}$ florescence emissions from the ${ }^{237} \mathrm{~Np}$ decay product. There are also a cluster of escape peaks between $30-40 \mathrm{keV}$ that are the result of the generation of $\mathrm{Cd} \& \mathrm{Te}$ florescence X-rays at energies between $23-32 \mathrm{keV}$. A $60 \mathrm{keV} \gamma$-ray interacting in the detector causes the $\mathrm{CdTe}$ to florescence and these X-rays, due to their relatively large mean free path, are able to either leave the detector volume or are absorbed beyond the nearest neighbour pixels and are uncorrected; the remaining photon energy is observed as an escape peak. The performance of the detector system under flat field illumination with higher energy $\gamma$-rays from the ${ }^{57} \mathrm{Co}$ can be seen in Figure 3 (right); the global FWHM at $122 \mathrm{keV}$ is also $1.2 \mathrm{keV}$. As with the ${ }^{241} \mathrm{Am}$ source a number of additional $\mathrm{Cd}$ and $\mathrm{Te}$ escape and florescence peaks can be observed at lower energies.

\section{HEXITEC - X-Ray Florescence Imaging at Synchrotrons}

For the majority of experiments carried out at synchrotrons using the HEXITEC system, a pinhole camera setup has been used to provide geometric magnification. This technique has been applied to both X-ray florescence and X-ray diffraction imaging modalities as well as simultaneous measurements of both [2]-[4]. The following results are an example of a simple XRF measurement that was made as part of the setup and alignment of a HEXITEC system on the ID19 beamline at ESRF. The detector system was placed inside a steel enclosure with a sensor to pinhole distance of $320 \mathrm{~mm}$ and a pinhole to sample distance of $36 \mathrm{~mm}$ providing a geometric magnification of $\sim \times 10$. The pinhole was $1 \mathrm{~mm}$ thick and produced from tungsten with an aperture size of $50 \mu \mathrm{m}$. Under these conditions the field of view at the sensor was $2 \mathrm{~mm} \times 2 \mathrm{~mm}$ with a spatial resolution of $25 \mu \mathrm{m}$ per pixel. The alignment sample was a $3.05 \mathrm{~mm}$ diameter gold transmission electron microscope 200 mesh grid ( $74 \mu \mathrm{m}$ grid pitch) with alphabetic identification for each set of nine grid squares (Agar Scientific - AGG2762A). A $5 \mathrm{~mm} \times 5 \mathrm{~mm}$ beam of polychromatic high energy X-rays was used to irradiate the entire grid. The HEXITEC system was positioned at $90^{\circ}$ to the sample to collect the florescence X-rays produced by the sample.

Figure 5 (left) shows examples of the average spectrum measured in regions of the field of view that correspond to the gold TEM grid and to those that represent the background. In the gold grid region there are clear spectral lines that coincide with the $\mathrm{K}_{\alpha 1}, \mathrm{~K}_{\alpha 2}, \mathrm{~K}_{\beta 1}$ and $\mathrm{K}_{\beta 2}$ emission lines at 68.8, 67.0, 78.0 and $80.2 \mathrm{keV}$ respectively as well as the much lower energy $\mathrm{L}_{\alpha 1}$ and $\mathrm{L}_{\beta 1}$ at 9.7 and $11.4 \mathrm{keV}$. The spectrum of the region of the field of view corresponding to the background also shows a number of prominent lines. These lines correspond to the $\mathrm{K}_{\alpha 1}, \mathrm{~K}_{\alpha 2}, \mathrm{~K}_{\beta 1}$ and $\mathrm{K}_{\beta 2}$ emission lines of tungsten at 
59.3, 58.0, 67.2 and 69.1 keV respectively as well as the much lower energy $L_{\alpha 1}$ and $L_{\beta 1}$ at 8.4 and 9.7 $\mathrm{keV}$. If a background subtraction is completed and the number of $\mathrm{K}_{\alpha}$ and $\mathrm{K}_{\beta}$ events measured in each pixel plotted, it is possible to produce an image of the gold TEM grid as seen in Figure 5 (right). As the HEXITEC system was positioned at a $45^{\circ}$ angle to the sample, the image is distorted in the horizontal direction due to an effective anisotropic pixel resolution. To correct for this distortion the raw image was re-sampled using interpolation to increase the number of pixels in the horizontal direction to achieve an isotropic pixel resolution. The processed image clearly shows the mesh of the grid and it's also possible to make out the letterforms within these grids. This is a simple demonstration of how the HEXITEC system can be used to perform mapping of multiple high Z elements on synchrotron beamlines [2].
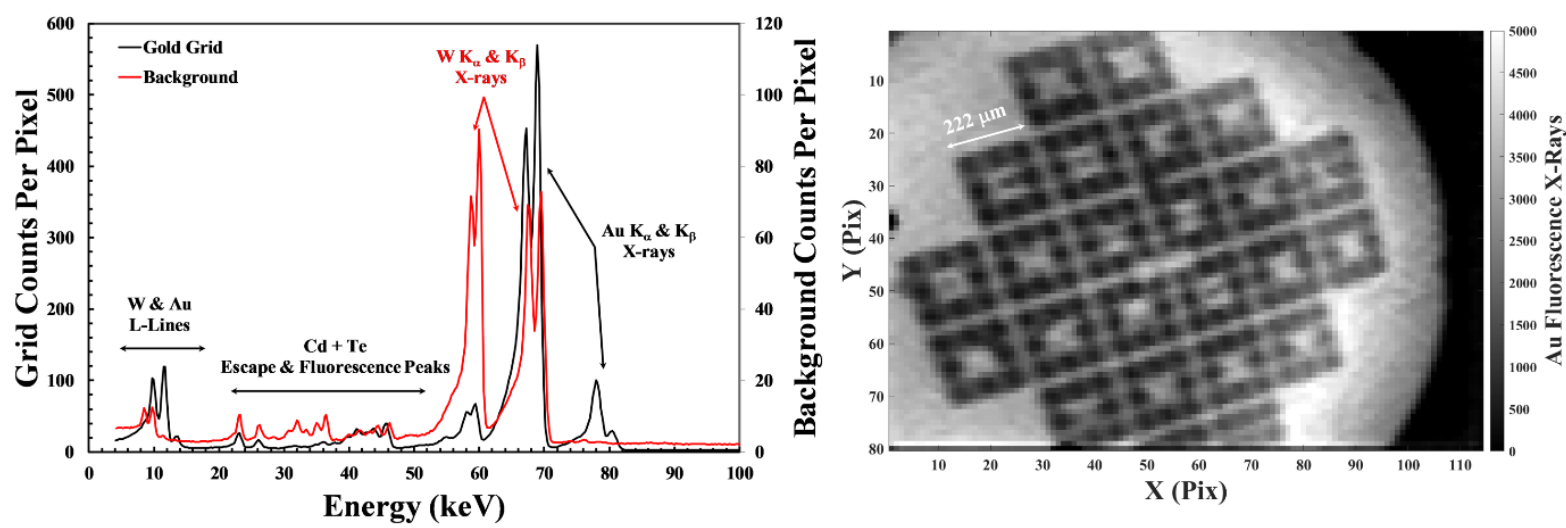

Figure 5: (left) the average spectrum per pixel measured by the HEXITEC system in regions coinciding with a TEM grid (black) and the background (red). (Right) An image of the intensity of gold $\mathrm{K}_{\alpha}$ and $\mathrm{K}_{\beta}$ emissions in the field of view of the sensor.

\section{Conclusion}

In this article the performance of the HEXITEC high energy X-ray spectroscopic imaging detector system has been demonstrated. The system is capable of measuring both the energy and position of photons interacting within the detector system producing X-ray spectra in each $250 \mu \mathrm{m}$ pitch pixel of an $80 \times 80$ array. In the energy range $2-200 \mathrm{keV}$ the system has an energy resolution (FWHM) of $800 \mathrm{eV}$ and is able to operate at room temperature. The unique features of this system enable the measurement of mechanical, structural or chemical information from thick samples as well as determining the chemical composition of samples containing multiple high $\mathrm{Z}$ materials. While the current iteration of the detector system is now in use at beam lines, a roadmap for STFC's continued development of this technology is in place. In the short term, the aim is to improve the efficiency of the system at energies $>150 \mathrm{keV}$ through the use of thick cadmium zinc telluride sensor material [18] without compromising on the spectroscopic performance of the system. In the longer term, a new generation of the technology will be developed that delivers spectroscopic imaging at frame rates of up to $1 \mathrm{MHz}$ which will meet the needs of future diffraction limited storage ring synchrotrons.

\section{Acknowledgements}

The authors would like to thank the STFC Centre for Instrumentation and the Engineering \& Physical Sciences Research Council (UK) grant numbers EP/D048737/1 \& EP/H046577/1 for the funding of the development and support of the HEXITEC technology. We would also like to thank T. Connolly from the I-12 beam line at the Diamond Light Source, and A. Lui from the University of Oxford and A. Rack of the I-19 beam line at the ESRF for their contribution to the synchrotron imaging results. HEXITEC systems are available from Quantum Detectors, UK. (https://quantumdetectors.com/n/products/hexitec/) 


\section{References}

[1] P. Seller et al, "Pixellated Cd(Zn)Te high-energy X-ray instrument," 2011 JINST 6 C12009. $(\underline{10.1088 / 1748-0221 / 6 / 12 / C 12009)}$

[2] E. Liotti et al, "Mapping of multi-elements during melting and solidification using synchrotron Xrays and pixel-based spectroscopy," 2015 Sci. Rep. 5 15988. (10.1038/srep15988)

[3] C. Egan et al, "3D chemical imaging in the laboratory by hyperspectral X-ray computed tomography,” 2015 Sci. Rep. 5 15979. (10.1038/srep15979)

[4] R. Rakowski et al, "Single-shot structural analysis by high-energy X-ray diffraction using an ultrashort all-optical source," 2017 Sci. Rep. 7 16603. (10.1038/s41598-017-16477-0)

[5] A. Hillier et al, "Element Specific Imaging Using Muonic X-rays," 2018 JPS Conf. Proc. 21 011042. (10.7566/JPSCP.21.011042)

[6] W. H. Baumgartner et al, "The HEXITEC Hard X-Ray Pixelated CdTe Imager for Fast Solar Observations," 2016 Proc. SPIE 9915, High Energy, Optical, and Infrared Detectors for Astronomy VII, 99151D. (10.1117/12.2234655)

[7] J. W. Scuffham et al, "Imaging of Ra-223 with a small-pixel CdTe detector," 2015 JINST 10 C01029 (10.1088/1748-0221/10/01/C01029)

[8] R. Moss et al, "miniPixD: a compact sample analysis system which combines X-ray imaging and diffraction," 2017 JINST 12 P02001. (10.1088/1748-0221/12/02/P02001)

[9] L. Jones et al, "HEXITEC ASIC - a pixelated readout chip for CZT detectors," 2009 Nuc. Inst. Meth. A. 604 pg. 34 - 37. (10.1016/j.nima.2009.01.046)

[10] H. Shiraki et al, "Improvement of the Productivity in the THM Growth of CdTe Single Crystal as Nuclear Radiation Detector,” IEEE Trans. Nuc. Sci. 201057 pg. 395 - 399.

$(\underline{10.1109 / T N S .2009 .2035316)}$

[11] A. Schneider et al, "Interconnect and bonding techniques for pixelated X-ray and gamma-ray detectors," 2015 JINST 10 C02010 (10.1088/1748-0221/10/02/C02010)

[12] M. Wilson et al, "A $10 \mathrm{~cm} \times 10 \mathrm{~cm}$ CdTe Spectroscopic Imaging Detector based on the HEXITEC ASIC,” 2015 JINST 10 P10011 (10.1088/1748-0221/10/10/P10011)

[13] A. Owens and A. Peacock, "Compound semiconductor radiation detectors," 2004 Nuc. Inst. Meth. A. 531 pg. 18-37. (10.1016/j.nima.2004.05.071)

[14] Zhong He, "Review of the Shockley-Ramo theorem and its application in semiconductor gammaray detectors," 2001 Nuc. Inst. Meth. A. 463 pg. 250 - 267. (10.1016/S0168-9002(01)00223-6)

[15] M. C. Veale et al, "Measurements of charge sharing in small pixel CdTe detectors," 2014 Nuc. Inst. Meth. A. 767 pg. 218 - 226. (10.1016/j.nima.2014.08.036)

[16] D. D. Duarte et al, "Edge effects in a small pixel CdTe for X-ray imaging," 2013 JINST 8 P10018. (10.1088/1748-0221/8/10/P10018)

[17] E. Fröjdh et al, "Depth of interaction and bias voltage depenence of the spectral response in a pixellated CdTe detector operating in time-over-threshold mode subjected to monochromatic X-rays," 2012 JINST 7 C03002. (10.1088/1748-0221/7/03/C03002) 
[18] M. C. Veale et al, "Synchrotron characterisation of non-uniformities in a small pixel cadmium zinc telluride imaging detector," 2013 Nuc. Inst. Meth. A. 729 pg. $265-272$.

$(\underline{10.1016 / j . n i m a .2013 .07 .054)}$ 